\title{
A new grading system focusing on neurological outcomes for brain metastases treated with stereotactic radiosurgery: the modified Basic Score for Brain Metastases
}

\author{
Clinical article
}

\author{
Toru Serizawa, M.D., Ph.D., ${ }^{1}$ Yoshinori Higuchi, M.D., Ph.D., ${ }^{2}$ \\ Osamu Nagano, M.D., Ph.D., ${ }^{3}$ Shinji Matsuda, M.D., Ph.D., Junichi Ono, M.D., Ph.D., ${ }^{3}$ \\ Naokatsu Saeki, M.D., Ph.D., ${ }^{2}$ Tatsuo Hirai, M.D., Ph.D., ${ }^{4}$ \\ Akifumi Miyakawa, M.D., Ph.D., 5 and Yuta Shibamoto, M.D., D.M.Sc. 5 \\ ${ }^{1}$ Tokyo Gamma Unit Center, Tsukiji Neurological Clinic, Tokyo; ${ }^{2}$ Department of Cerebral and Neurological \\ Surgery, Chiba University Graduate School of Medicine, Chiba; ${ }^{3}$ Gamma Knife House, Chiba \\ Cardiovascular Center, Ichihara; ${ }^{4}$ Heisei Gamma Unit Center, Fujieda Heisei Memorial Hospital, Fujieda; \\ and ${ }^{5}$ Department of Radiology, Graduate School of Medical Sciences and Medical School, Nagoya City \\ University, Nagoya, Japan
}

\begin{abstract}
Object. The Basic Score for Brain Metastases (BSBM) proposed by Lorenzoni and colleagues is one of the best grading systems for predicting survival periods after stereotactic radiosurgery (SRS) for brain metastases. However, it includes no brain factors and cannot predict neurological outcomes, such as preservation of neurological function and prevention of neurological death. Herein, the authors propose a modified BSBM, adding 4 brain factors to the original BSBM, enabling prediction of neurological outcomes, as well as of overall survival, in patients undergoing SRS.

Methods. To serve as neurological prognostic scores (NPSs), the authors scored 4 significant brain factors for both preservation of neurological function (qualitative survival) and prevention of neurological death (neurological survival) as 0 or 1 as described in the following: $>10$ brain tumors $=0$ or $\leq 10=1$, total tumor volume $>15 \mathrm{~cm}^{3}=0$ or $\leq 15 \mathrm{~cm}^{3}=1$, MRI findings of localized meningeal dissemination (yes $=0$ or no $=1$ ), and neurological symptoms (yes $=0$ or no $=1)$. According to the sum of NPSs, patients were classified into 2 subgroups: Subgroup A with a total NPS of 3 or 4 and Subgroup B with an NPS of 0,1 , or 2. The authors defined the modified BSBM according to the NPS subgroup classification applied to the original BSBM groups. The validity of this modified BSBM in 2838 consecutive patients with brain metastases treated with SRS was verified.

Results. Patients included 1868 with cancer of the lung (including 1604 with non-small cell lung cancer), 355 of the gastrointestinal tract, 305 of the breast, 176 of the urogenital tract, and 134 with other cancers. Subgroup A had 2089 patients and Subgroup B 749. Median overall survival times were 2.6 months in BSBM 0 (382 patients), 5.7 in BSBM 1 (1143), 11.4 in BSBM 2 (1011) and 21.7 in BSBM 3 (302), and pairwise differences between the BSBM groups were statistically significant (all $\mathrm{p}<0.0001$ ). One-year qualitative survival rates were $64.6 \%$ (modified BSBM 0A, 204 patients), 45.0\% (0B, 178), 82.5\% (1A, 825), 63.3\% (1B, 318), 86.4\% (2A, 792), 73.7\% (2B, 219), 91.4\% $(3 \mathrm{~A}, 268)$, and $73.5 \%(3 \mathrm{~B}, 34)$. One-year neurological survival rates were $82.6 \%(0 \mathrm{~A}), 52.4 \%(0 \mathrm{~B}), 90.5 \%(1 \mathrm{~A})$, $78.1 \%$ (1B), $91.1 \%(2 \mathrm{~A}), 83.2 \%(2 \mathrm{~B}), 93.9 \%$ (3A), and 76.3\% (3B), where A and B identify the subgroup. Statistically significant differences in both qualitative and neurological survivals between Subgroups A and B were detected in all BSBM groups.

Conclusions. The authors' new index, the modified BSBM, was found to be excellent for predicting neurological outcomes, independently of life expectancy, in SRS-treated patients with brain metastases. (http://thejns.org/doi/abs/10.3171/2014.7.GKS14980)
\end{abstract}

\section{KeY Words - brain metastases prevention of neurological death \\ - preservation of neurological function - stereotactic radiosurgery - Gamma Knife surgery}

\footnotetext{
Abbreviations used in this paper: $\mathrm{ADL}=$ activity of daily living; $\mathrm{BSBM}=$ Basic Score for Brain Metastases; GKS = Gamma Knife surgery; GPA = graded prognostic assessment; KPS = Karnofsky performance status; $\mathrm{MD}=$ meningeal dissemination; NPS = neurological prognostic score; RPA = recursive partitioning analysis; $\mathrm{SIR}=$ score index for stereotactic radiosurgery; SRS = stereotactic radiosurgery; $\mathrm{WBRT}=$ whole-brain radiation therapy.
}

$\mathrm{N}$ EUROLOGICAL outcomes, such as preservation of neurological function ${ }^{16}$ and prevention of neurological death, are regarded as the ideal end points for evaluating treatment results for patients with brain metastases. However, previous reports on grading systems for brain metastasis patients, such as recursive partitioning analysis (Gaspar et al., ${ }^{3}$ 1997), score index for 
stereotactic radiosurgery (SIR; Weltman et al., ${ }^{18}$ 2000), Basic Score for Brain Metastases (BSBM; Lorenzoni et al., ${ }^{8}$ 2004), graded prognostic assessment (GPA; Sperduto et al. ${ }^{17}$ 2008), and modified RPA (Yamamoto et al., ${ }^{21}$ 2013) are based only on overall survival. Among these grading systems, the BSBM is one of the best for predicting life expectancy by using the 3 systemic prognostic factors: Karnofsky Performance Scale (KPS) score, ${ }^{7}$ control of primary tumor, and presence of extracranial metastases. However, the BSBM includes no brain factors and cannot predict neurological outcomes. Herein, we propose a modified BSBM, adding 4 brain factors to the original BSBM, enabling prediction of neurological outcomes (both preservation of neurological function and prevention of neurological death), independently of life expectancy, in patients undergoing Gamma Knife surgery (GKS).

\section{Methods}

In total, 2838 patients were included in the analysis. The database consisted of 2 patient cohorts studied retrospectively after institutional review board approval: the Chiba series (1716 consecutive patients, January 1998 through March 2008, Chiba Cardiovascular Center) and the Tokyo series (1122 patients, April 2008 through December 2013, Tsukiji Neurological Clinic). All aspects of patient selection, dose planning, dose selection, performing GKS, and collecting follow-up data were undertaken by the first author (T.S.).

During the 16-year study period, all patients were treated according to the same indications and protocol. Our major indication criteria were the following: 1) tumor number up to 25, 2) no MRI findings of nonlocalized meningeal dissemination (MD), 3) maximum tumor diameter less than $40 \mathrm{~mm}$, 4) total tumor volume less than $25 \mathrm{~cm}^{3}$, and 5) KPS score greater than 70\%. However, we applied these indications with flexibility, especially for patients with special circumstances, such as contraindications for whole-brain radiation therapy (WBRT) and craniotomy or expectation of a good response to smallmolecule targeting agents. Patients with KPS scores less than $70 \%$ due to focal neurological signs and symptoms and whose performance status was expected to improve after GKS were included.

Our GKS treatment protocol was reported previously. ${ }^{10,12-14}$ In brief, all lesions were irradiated with GKS without upfront WBRT at initial treatment. In 293 patients $(10.3 \%)$ with tumor volumes exceeding $10 \mathrm{~cm}^{3}$, staged SRS using the Gamma Knife, ${ }^{5,2}$ was chosen, and in 89 (3.1\%) with a total tumor volume exceeding $15 \mathrm{~cm}^{3}$, tumor numbers greater than 25 , or both, the radiosurgical procedures were divided into 2 or 3 sessions, to keep total skull absorption energy below $10 \mathrm{~J}$, thereby preventing acute brain swelling. ${ }^{13}$ New distant lesions, detected by gadoliniumenhanced MRI performed every 2-3 months, were treated mainly with GKS (1236 patients, $43.6 \%$ ), but sometimes with WBRT $(140,5.0 \%)$ if cerebral and/or nonlocalized MD was detected. The treatment strategy was explained in detail to each patient and written informed consent was obtained from all patients before GKS.
A stereotactic coordinate frame (Leksell G frame; Elekta AB) was applied to patients under local anesthesia supplemented with adequate sedation. For target coordinate determination and dose planning, stereotactic gadolinium-enhanced, T1-weighted axial MRI, with a slice thickness of $2 \mathrm{~mm}$ and multiple slices covering the entire brain, was used. For dose planning, the Leksell GammaPlan (Elekta) was used. Before October 2003, GKS was performed using a Leksell GK model B (659 patients, January 1988-September 2003; Elekta). From October 2003 to November 2011, we used a Leksell GK model C (1767 patients; Elekta) and from December 2011 to December 2013, we used a Perfexion Gamma Knife (412 patients; Elekta). The standard prescribed dose at the tumor periphery of 20 Gy was changed by \pm 2 Gy depending on tumor pathology, physical status, tumor location, tumor volume, extracranial disease status, and so on.

All data were analyzed according to the intentionto-treat principle. For all patients, the causes of impaired activities of daily living (ADLs) and deaths were classified into systemic or neurological by referring physicians. If the referring physician could not make this assessment, the causes were regarded as neurological. The intervals from the date of GKS until the dates of death (overall survival), systemic death (systemic death-free survival), loss of ADLs independence due to neurological causes (qualitative survival), and neurological death (neurological survival) were estimated with Kaplan-Meier curves. ${ }^{6}$ Loss of ADLs independence was defined as a KPS score of $<70 \% .1,1,11,15,20$ The differences between 2 adjacent classes were compared using the Wilcoxon test. Prognostic values of the following 15 dichotomized covariates for qualitative and neurological survivals were obtained with the Cox proportional hazards model: ${ }^{2}$ age $(<65$ years vs $\geq 65$ years), sex (male vs female), KPS score ( $<80 \%$ vs $\geq 80 \%$ ), control of primary tumor (yes vs no), extracranial metastases (yes vs no), organ site of primary cancer (lung/breast vs nonlung/breast), number of brain tumors ( $\leq 10 \mathrm{vs}>10)$, total tumor volume $\left(\leq 15 \mathrm{~cm}^{3} \mathrm{vs}>15 \mathrm{~cm}^{3}\right)$, MRI findings of MD (yes vs no), craniotomy (yes vs no), chemotherapy (yes vs no), diagnostic lag (synchronous vs metachronous), prior WBRT (yes vs no), maximum tumor diameter ( $\geq 25 \mathrm{~mm}$ vs $<25 \mathrm{~mm}$ ), and neurological symptoms (yes vs no). A p value $<0.05$ was considered statistically significant. All statistical analyses were performed using the JMP software program, version 9.0.3 (SAS Institute Inc.).

\section{Results}

At the end of April 2014, 369 (13.0\%) patients were still alive and the remaining $2469(87.0 \%)$ were confirmed to have died. Table 1 summarizes the characteristics of the patients in this study. The median times were 7.8 months for overall survival and 9.0 months for systemic death-free survival. Figure 1 shows overall survival curves according to the original BSBM. Median overall survival times were 2.6 months in BSBM 0 (382 patients), 5.7 in BSBM 1 (1143), 11.4 in BSBM 2 (1011), and 21.7 in BSBM 3 (302). There were significant differences between all pairs of adjacent groups (all $\mathrm{p}<0.0001$ ). Figure 2 shows systemic death- 
TABLE 1: Characteristics of the patients with brain metastases in this study

\begin{tabular}{|c|c|}
\hline Variables & No. of Patients (\%)* \\
\hline all patients & $2838(100)$ \\
\hline \multicolumn{2}{|l|}{ age } \\
\hline$\geq 65$ yrs & $1484(52.3)$ \\
\hline$<65$ yrs & $1354(47.7)$ \\
\hline \multicolumn{2}{|l|}{ sex } \\
\hline male & $1692(59.6)$ \\
\hline female & $1146(40.4)$ \\
\hline \multicolumn{2}{|l|}{ KPS score (\%) } \\
\hline 100 & $1133(39.9)$ \\
\hline 90 & $587(20.7)$ \\
\hline 80 & $446(15.7)$ \\
\hline 70 & $296(10.4)$ \\
\hline 60 & $315(11.1)$ \\
\hline 50 & $61(2.1)$ \\
\hline \multicolumn{2}{|l|}{ controlled primary cancer } \\
\hline yes & $881(31.0)$ \\
\hline no & $1957(69.0)$ \\
\hline \multicolumn{2}{|l|}{ extracranial metastasis } \\
\hline yes & $1814(63.9)$ \\
\hline no & $1024(36.1)$ \\
\hline \multicolumn{2}{|l|}{ primary cancer site } \\
\hline lung & $1868(65.8)$ \\
\hline gastrointestinal tract & $355(12.5)$ \\
\hline breast & $305(10.7)$ \\
\hline urogenital & $176(6.2)$ \\
\hline other & $134(4.7)$ \\
\hline \multicolumn{2}{|c|}{$\begin{array}{l}\text { diagnostic lag btwn primary cancer \& brain } \\
\text { metastases }\end{array}$} \\
\hline synchronous & $1005(35.4)$ \\
\hline metachronous & $1833(64.6)$ \\
\hline prior craniotomy & 375 (13.2) \\
\hline prior chemotherapy & $1670(58.8)$ \\
\hline prior WBRT & $214(7.5)$ \\
\hline \multicolumn{2}{|l|}{ no. of brain tumors } \\
\hline 1 & 799 (28.2) \\
\hline $2-4$ & $931(32.8)$ \\
\hline $5-10$ & $623(21.9)$ \\
\hline $11-25$ & $375(13.2)$ \\
\hline$>25$ & $110(3.9)$ \\
\hline \multicolumn{2}{|l|}{ tumor diameter† } \\
\hline$\geq 25 \mathrm{~mm}$ & $1045(36.8)$ \\
\hline$<25 \mathrm{~mm}$ & 1793 (63.2) \\
\hline \multicolumn{2}{|l|}{ total tumor vol } \\
\hline$>15 \mathrm{~cm}^{3}$ & $520(18.3)$ \\
\hline$\leq 15 \mathrm{~cm}^{3}$ & $2318(81.7)$ \\
\hline MRI findings of MD & $323(11.4)$ \\
\hline
\end{tabular}

(continued)
TABLE 1: Characteristics of the patients with brain metastases in this study (continued)

\begin{tabular}{lc}
\hline \multicolumn{1}{c}{ Variables } & No. of Patients $(\%)^{*}$ \\
\hline neurological symptoms & \\
yes & $1324(46.7)$ \\
no & $1514(53.3)$ \\
staged GKS procedures & \\
yes & $293(10.3)$ \\
no & $2545(89.7)$ \\
\hline
\end{tabular}

* The median age of all patients was 65 years (range 7-94 years).

$\dagger$ The median tumor diameter was $20.8 \mathrm{~mm}$ (range $4.0-83.1 \mathrm{~mm}$ ).

$\ddagger$ The median tumor volume was $4.4 \mathrm{~cm}^{3}$ (range $\left.0.1-100 \mathrm{~cm}^{3}\right)$.

free survival curves according to the original BSBM. Median systemic death-free survival times were 2.9 months in BSBM 0, 6.0 in BSBM 1, 13.2 in BSBM 2, and 31.6 in BSBM 3. The differences between all pairs of adjacent groups were statistically significant (all $\mathrm{p}<0.0001$ ).

In univariate analysis, significant prognostic factors for poor qualitative survival were a KPS score $<80 \%$, active primary tumor, active extracranial metastases, nonlung/breast cancer, prior craniotomy, no chemotherapy, prior WBRT, maximum tumor diameter $\geq 25 \mathrm{~mm},>10$ brain tumors, total tumor volume $>15 \mathrm{~cm}^{3}$, MRI findings of MD, and the presence of neurological symptoms (Table 2). Significant prognostic factors for poor neurological survival were the same as those for qualitative survival (Table 3). In multivariate analysis, $>10$ brain tumors, $>15 \mathrm{~cm}^{3}$ total tumor volume, MRI findings of localized MD, and the presence of neurological symptoms were verified to be the most statistically significant combination of factors for both qualitative and neurological survivals (Table 4).

To obtain NPSs, we scored the following 4 significant brain factors as 0 or 1: number of brain tumors $(>10=0$ or 10 or less $=1)$, total tumor volume $\left(>15 \mathrm{~cm}^{3}=0\right.$ or $\leq$ $15 \mathrm{~cm}^{3}=1$ ), MRI findings of MD (yes $=0$ or no $=1$ ), and neurological symptoms (yes $=0$ or no $=1)$. According

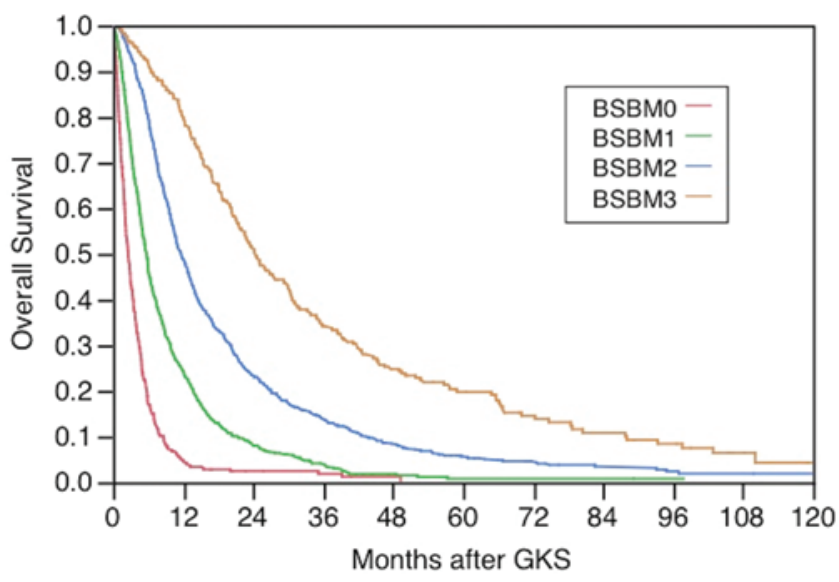

FIG. 1. Overall survival curves according to the original BSBM system. Median overall survival times were 2.6 months in BSBM 0 (382 patients), 5.7 in BSBM 1 (1143), 11.4 in BSBM 2 (1011), and 21.7 in BSBM 3 (302). Statistically significant differences were detected between each pair of adjacent groups (all $p<0.0001)$. 


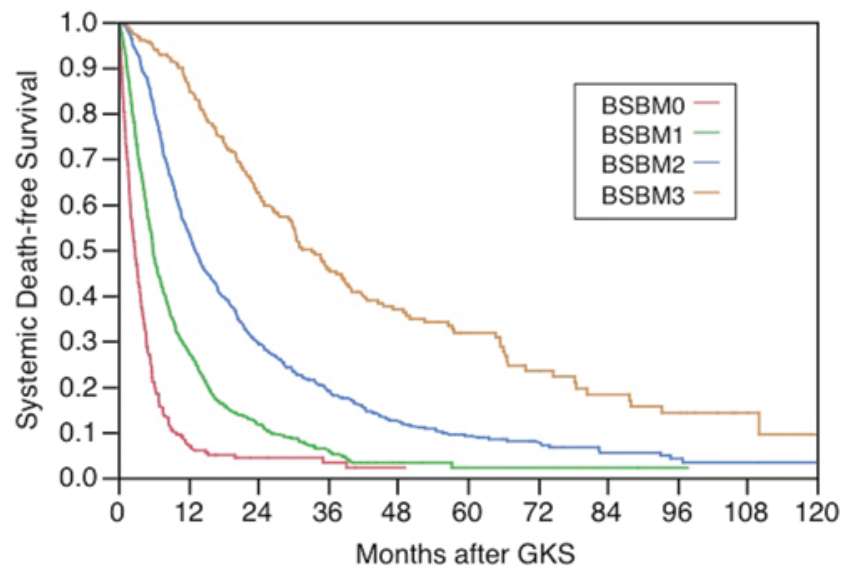

FIG. 2. Systemic death-free survival curves according to the original BSBM system. Median systemic death-free survival times were 2.9 months in BSBM 0 (382 patients), 6.0 in BSBM 1 (1143), 13.2 in BSBM 2 (1011), and 31.6 in BSBM 3 (302). The differences between all pairs of adjacent groups were significant (all $p<0.0001$ ).

to the NPS sum, we classified patients into 2 subgroups: Subgroup A with an NPS of 3 or 4 and Subgroup B with an NPS of 0,1 , or $2 ; 2089$ patients $(73.6 \%)$ were in Subgroup A and 749 (26.4\%) in Subgroup B. Figure 3 shows qualitative and neurological survival curves comparing the NPS Subgroups A and B. Qualitative survival rates at 1 year were 85.5\% in Subgroup A and 65.6\% in Subgroup $\mathrm{B}$, and neurological survival rates at 1 year were $91.3 \%$ in Subgroup A and 76.8\% in Subgroup B. We noted significant differences between Subgroups A and B in both qualitative and neurological survivals (both $\mathrm{p}<0.0001$ ). We defined the modified BSBM according to NPS subgroup classifications applied to each original BSBM group, consisting of $0 \mathrm{~A}$ (204 patients), 0B (178), 1A (825), 1B (318), 2A (792), 2B (219), 3A (268), and 3B (34), where $A$ and $B$ refer to the respective subgroup (Table 5).

TABLE 2: Univariate analysis of the value of covariates for predicting qualitative survival ${ }^{*}$

\begin{tabular}{lcr}
\hline \multicolumn{1}{c}{ Factor for Prognosis } & HR $(95 \% \mathrm{Cl})$ & p Value \\
\hline age $\geq 65$ yrs & $1.06(0.90-1.26)$ & 0.5207 \\
male sex & $1.17(0.98-1.39)$ & 0.0809 \\
KPS score $<80 \%$ & $2.18(1.76-2.67)$ & $<0.0001$ \\
no control of primary tumor & $1.20(1.01-1.44)$ & 0.0430 \\
extracranial metastases & $1.54(1.29-1.85)$ & $<0.0001$ \\
nonlung/breast tumor at primary site & $1.48(1.20-1.81)$ & 0.0003 \\
metachronous diagnostic lag & $1.01(0.87-1.22)$ & 0.8788 \\
craniotomy & $1.34(1.06-1.68)$ & 0.0144 \\
no chemotherapy & $1.34(1.13-1.59)$ & 0.0010 \\
prior WBRT & $1.73(1.28-2.29)$ & 0.0005 \\
max tumor diameter $\geq 25$ mm & $1.92(1.61-2.29)$ & $<0.0001$ \\
no. of brain tumors $>10$ & $1.82(1.46-2.25)$ & $<0.0001$ \\
total tumor vol. $>15 \mathrm{~cm}^{3}$ & $2.32(1.94-2.90)$ & $<0.0001$ \\
MRI finding of MD & $2.40(1.88-2.91)$ & $<0.0001$ \\
neurological symptoms & $1.70(1.43-2.04)$ & $<0.0001$ \\
\hline
\end{tabular}

* $\mathrm{HR}=$ hazard ratio.
TABLE 3: Univariate analysis of the value of covariates for predicting neurological survival

\begin{tabular}{lcr}
\hline \multicolumn{1}{c}{ Factor for Prognosis } & $\mathrm{HR}(95 \% \mathrm{Cl})$ & $\mathrm{p} \mathrm{Value}$ \\
\hline age $\geq 65$ yrs & $1.11(0.90-1.36)$ & 0.3435 \\
male sex & $1.13(0.92-1.39)$ & 0.2402 \\
KPS score $<80 \%$ & $2.10(1.62-2.68)$ & $<0.0001$ \\
no control of primary tumor & $1.24(1.00-1.54)$ & 0.0491 \\
extracranial metastases & $1.55(1.25-1.92)$ & $<0.0001$ \\
nonlung/breast tumor at primary site & $1.47(1.14-1.87)$ & 0.0030 \\
metachronous diagnostic lag & $1.07(0.86-1.34)$ & 0.5225 \\
craniotomy & $1.41(1.07-1.83)$ & 0.0142 \\
no chemotherapy & $1.23(1.00-1.52)$ & 0.0461 \\
prior WBRT & $1.79(1.25-2.48)$ & 0.0019 \\
max tumor diameter $\geq 25$ mm & $2.04(1.65-2.50)$ & $<0.0001$ \\
no. of brain tumors $>10$ & $1.76(1.34-2.27)$ & $<0.0001$ \\
total tumor vol $>15 \mathrm{~cm}^{3}$ & $2.42(1.90-3.05)$ & $<0.0001$ \\
MRI finding of MD & $2.65(2.05-3.39)$ & $<0.0001$ \\
neurological symptoms & $1.65(1.34-2.04)$ & $<0.0001$ \\
\hline
\end{tabular}

Qualitative survival curves were used to compare the NPS subgroups (Fig. 4), and the neurological survival curves are shown for each BSBM group in Fig. 5 . One-year qualitative survival rates were $64.6 \%$ (modified BSBM 0A), $45.0 \%$ (0B), $82.5 \%$ (1A), 63.3\% (1B), $86.4 \%$ (2A), $73.7 \%$ (2B), $91.4 \%$ (3A), and $73.5 \%$ (3B). There were significant differences in qualitative survival among all $\mathrm{BSBM}$ groups (modified BSBM 0A vs $0 \mathrm{~B}, \mathrm{p}=0.0006$; modified BSBM 1A vs $1 \mathrm{~B}, \mathrm{p}<0.0001$; modified BSBM $2 \mathrm{~A}$ vs $2 \mathrm{~B}, \mathrm{p}<0.0001$; and modified BSBM $3 \mathrm{~A}$ vs $3 \mathrm{~B}, \mathrm{p}=$ $0.006)$. One-year neurological survival rates were $82.6 \%$ (modified BSBM 0A), 52.4\% (0B), 90.5\% (1A), 78.1\% (1B), $91.1 \%$ (2A), $83.2 \%$ (2B), $93.9 \%$ (3A), and $76.3 \%$ (3B). Statistically significant differences in neurological survival were also detected between the Subgroups A and B for BSBM $0(p=0.0001)$, BSBM $1(p<0.0001)$, BSBM $2(\mathrm{p}=0.0002)$, and BSBM $3(\mathrm{p}=0.0052)$.

\section{Discussion}

Five major indices, RPA, ${ }^{3}$ SIR $,{ }^{18} \mathrm{BSBM},{ }^{8} \mathrm{GPA},{ }^{17}$ and modified RPA, ${ }^{21}$ have been proposed in radiotherapy for brain metastases. Serizawa et al. ${ }^{9}$ reported on validity testing of these 5 indices in terms of overall and qualitative survivals in 2500 patients treated with GKS. All

TABLE 4: Multivariate analysis of the value of covariates for predicting qualitative and neurological survivals*

\begin{tabular}{lrrrrrr}
\hline \multirow{2}{*}{ Factor for Prognosis } & \multicolumn{2}{c}{ QS } & & \multicolumn{2}{c}{ NS } \\
\cline { 2 - 3 } \cline { 6 - 7 } & HR & p Value & & HR & p Value \\
\hline no. of brain tumors $>10$ & 1.53 & 0.0004 & & 1.43 & 0.0118 \\
total tumor vol $>15 \mathrm{~cm}^{3}$ & 1.94 & $<0.0001$ & & 2.01 & $<0.0001$ \\
MRI finding of localized MD & 2.05 & $<0.0001$ & & 2.36 & $<0.0001$ \\
neurological symptoms & 1.47 & $<0.0001$ & & 1.41 & 0.0020 \\
\hline
\end{tabular}

* NS = neurological survival; QS = qualitative survival. 

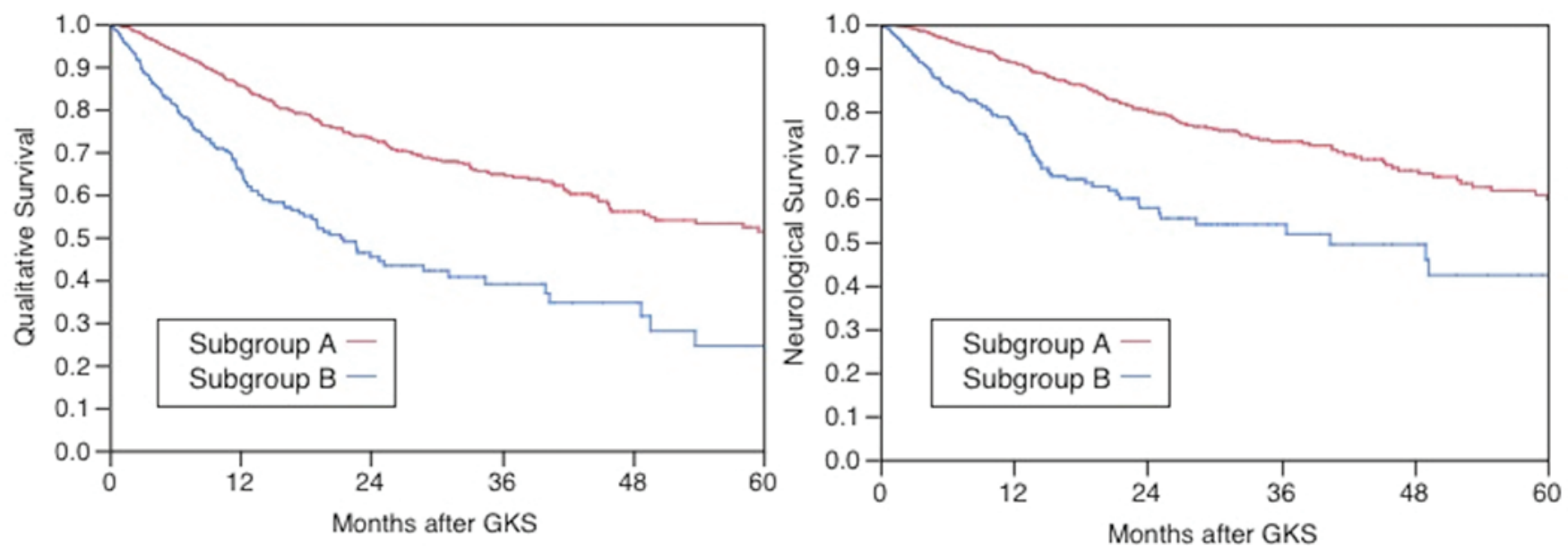

FIG. 3. Cumulative neurological deterioration-free survival (qualitative survival, left) and neurological death-free survival (neurological survival, right). Curves were compared between NPS Subgroups A and B. Statistically significant differences were detected between NPS Subgroups A (2089 patients) and B (749), in both qualitative and neurological survivals (both $p<0.0001)$.

5 grading systems were excellent for predicting life expectancy. The only grading system enabling prediction of both overall and qualitative survivals was the SIR, even though it is relatively complex with 5 items having 3 scores, and there were large discrepancies in patient numbers among groups. In the present study, the patients numbers in SIR 0-3 and 8-10 were $425(15.0 \%)$ and 241 (8.5\%), respectively, whereas that of SIR 4-7 was 2172 (76.5\%). Furthermore, SIR has the disadvantage that patient groups with poor overall survival (SIR 0-3) also had poor qualitative survival and vice versa. Thus, we have endeavored to establish a new grading system, the modified BSBM, which can predict neurological outcomes as well as life expectancy independently of each other.

The BSBM would appear to be one of the best indices, resulting in more even proportions of patient numbers with the highest level of statistical significance for differences in median overall survival, as well as for systemic death-free survival $(\mathrm{p}<0.0001$ for all pairs of adjacent classes). However, the BSBM considers only systemic and not neurological factors. Thus, it cannot predict neurological outcomes, such as preservation of neurological function and prevention of neurological death, which are regarded as the ideal end points in evaluating results of treatments of brain metastases. We therefore modified the original BSBM, adding 4 neurological prognostic factors, enabling us to predict neurological outcomes. The modified BSBM has 2 components. One is from the original BSBM evaluating 3 systemic factors: KPS score, primary tumor control, and extracranial metastases for predicting overall survival and systemic death-free survivals. The other involves 4 neurological factors: number of brain tumors, total tumor volume, MRI findings of MD, and neurological symptoms, which have all been verified as the most significant prognostic factors impacting both qualitative and neurological survivals in multivariate analyses and reflecting neurological outcomes. Therefore, this modified BSBM allows us to predict qualitative and neurological survivals, as well as overall and systemic death-free survivals, independently of other outcomes.

TABLE 5: Definition of the modified Basic Score for Brain Metastases

\begin{tabular}{ccc}
\hline NPS & NPS 0 & NPS 1 \\
\hline brain tumor no. & $>10$ & $\leq 10$ \\
total tumor vol & $>15 \mathrm{~cm}^{3}$ & $\leq 15 \mathrm{~cm}^{3}$ \\
MRI finding of MD & yes & no \\
neurological symptoms & yes & no \\
Classification of NPS Subgroups & Sum of NPS & No. of Patients \\
\hline A & 3,4 & 2089 \\
B & $0,1,2$ & 749 \\
\hline Modified BSBM & NPS Subgroup A & NPS Subgroup B \\
\hline 0 & OA (204) & OB (178) \\
1 & $1 \mathrm{~A}(825)$ & $1 \mathrm{~B}(318)$ \\
3 & $2 \mathrm{~A}(792)$ & $2 \mathrm{~B}(219)$ \\
\hline
\end{tabular}

\footnotetext{
* Subgroup classification and number of patients in each classification group.
} 
A. BSBM 0

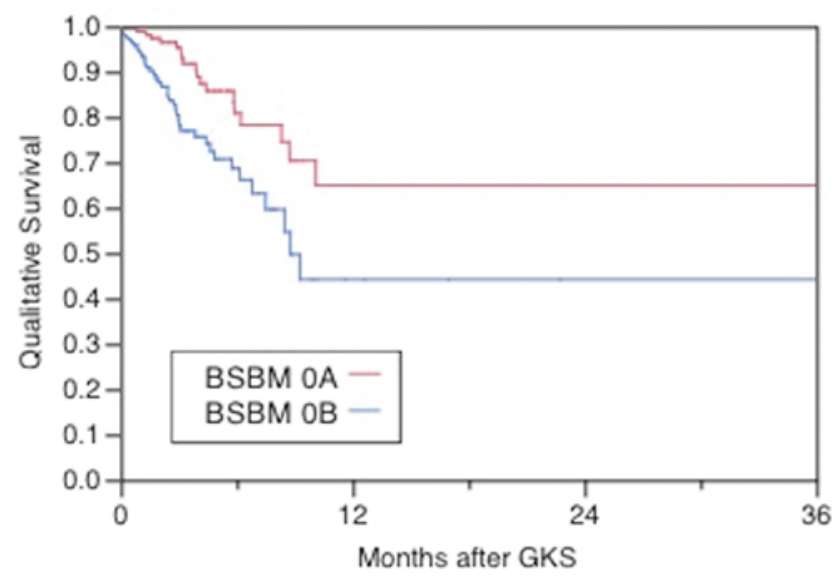

\section{BSBM 2}

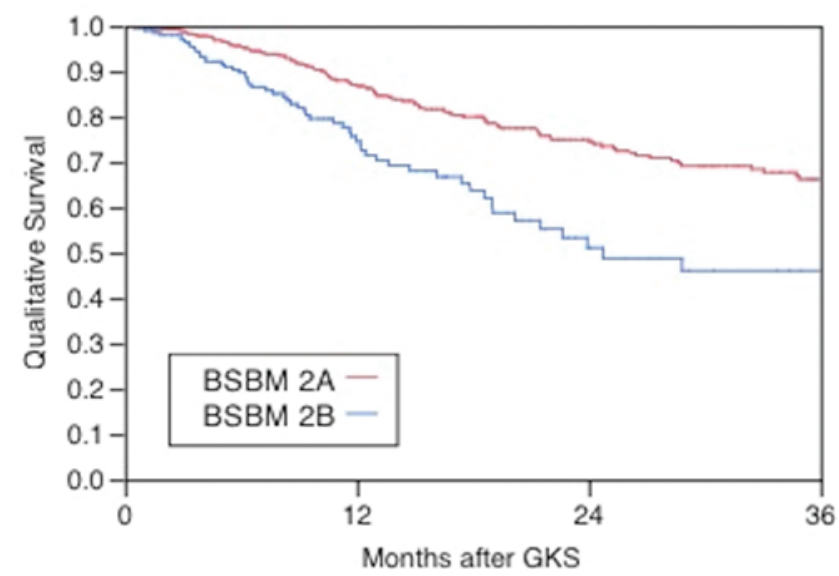

\section{B. BSBM 1}

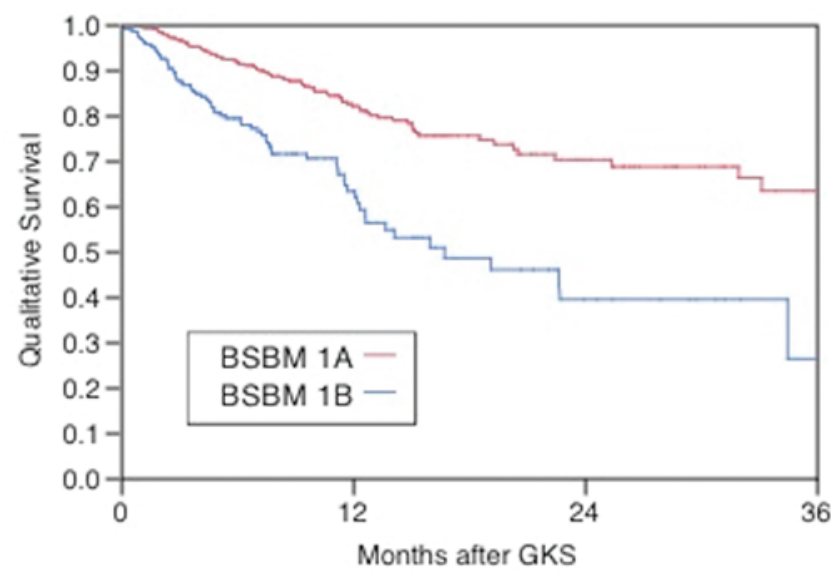

D. BSBM 3

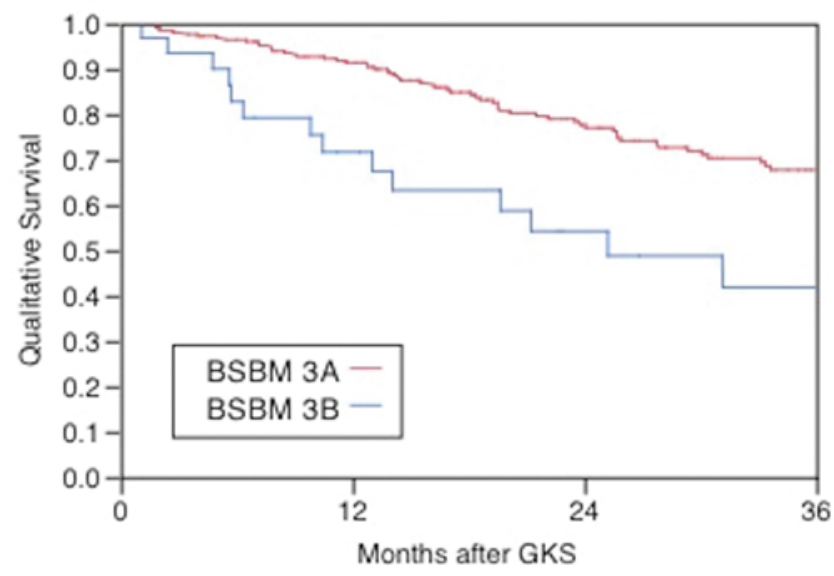

FIG. 4. Cumulative neurological deterioration-free survival (qualitative survival) curves allow comparison between NPS subgroups in each BSBM group. One-year qualitative survival rates were 64.6\% (modified BSBM 0A, 204 patients), 45.0\% (0B, 178), 82.5\% (1A, 825), 63.3\% (1B, 318), 86.4\% (2A, 792), 73.7\% (2B, 219), 91.4\% (3A, 268), and 73.5\% (3B, 34). Statistically significant differences in qualitative survival were detected between all $B S B M$ subgroups (BSBM $0 A$ vs $0 B, p=0.0006 ; 1 A$ vs $1 B$, $p<0.0001 ; 2 A$ vs $2 B, p<0.0001$; and $3 A$ vs $3 B, p=0.0006)$.

We selected 4 significant brain-related prognostic factors, including tumor number, total tumor volume, MRI findings of MD, and neurological symptoms on the basis of the results of multivariate analyses of qualitative and neurological survivals, and we scored these parameters as 0 or 1 to serve as NPSs. According to the sum of these NPSs, we divided patients into 2 subgroups: Subgroup A (containing patients with better neurological outcomes) with a score of 3 or 4 , and Subgroup B (those with poorer outcomes) with a score of 0,1 , or 2 for each of the original BSBM groups. The best neurological outcomes were observed in patients whose NPS totaled 4, whereas the worst were in those with a total NPS of 0 , as in the original BSBM (BSBM 0 has the shortest overall or systemic death-free survivals and BSBM 3 the longest). Choosing 4 brain factors seems to be relatively complex, but this selection made the NPS system extremely robust. For example, we can also apply the NPS system to the original GPA, ${ }^{16}$ demonstrating significant qualitative survival differences in each GPA class, as shown in Table 6.
We set the cutoff value at 10 for brain tumor number and at $15 \mathrm{~cm}^{3}$ for total tumor volume, corresponding to the JLGK0901 study inclusion criteria. ${ }^{21}$

Our results suggest that the patients in NPS Subgroup A would be good candidates for GKS alone, whereas those in NPS Subgroup B would be unlikely to benefit from GKS alone. However, patients in Subgroup B do not necessarily have contraindications for treatment with GKS alone if survival is anticipated to be very short. For example, in BSBM $0 \mathrm{~B}$ or 1B patients, even in those with $>10$ brain metastases and exhibiting neurological symptoms, GKS-alone treatment is an option because their life expectancy is too short for WBRT.

The qualitative and neurological survival rates at the median systemic death-free survival time are shown in Table 7. These results show satisfactory neurological outcomes in all groups except modified BSBM 3B. Patients in the modified BSBM 3B group have a longer life expectancy, but poorer neurological outcomes. For modified BSBM 3B patients with good KPS scores and controlled 


\section{A. BSBM 0}

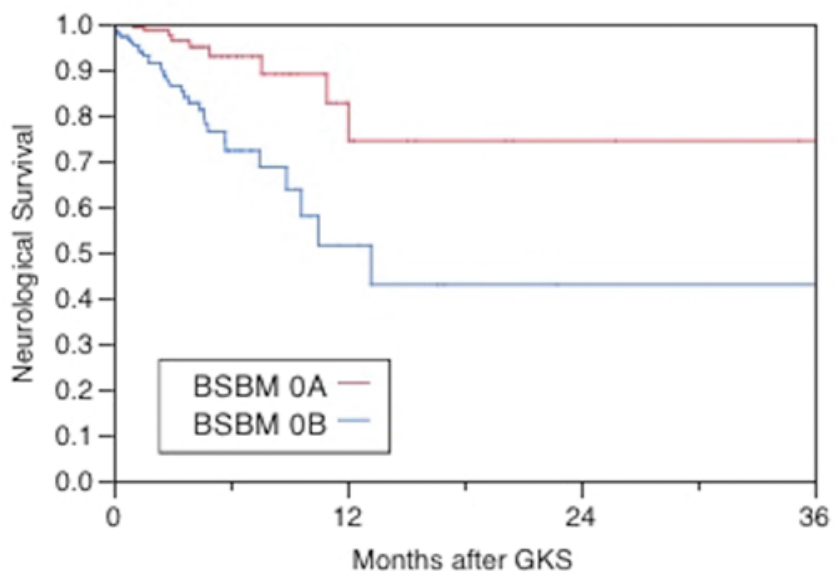

\section{BSBM 2}

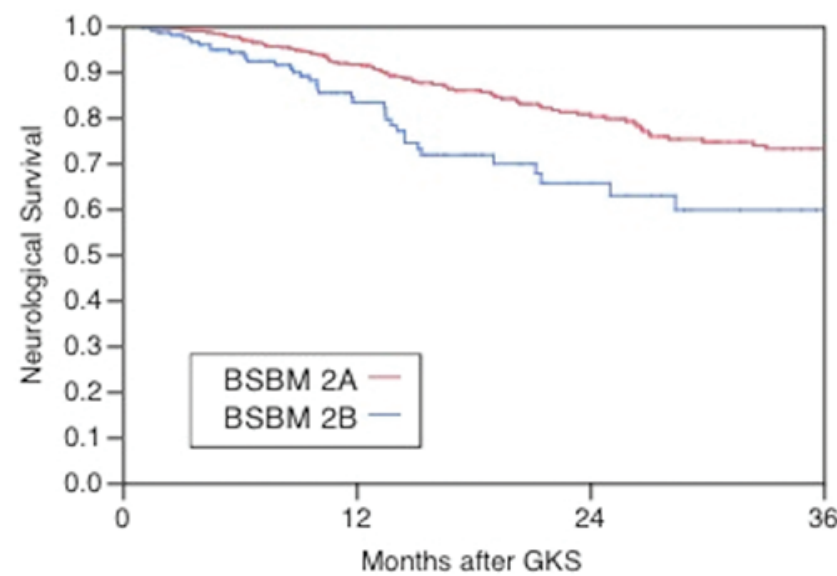

\section{B. BSBM 1}

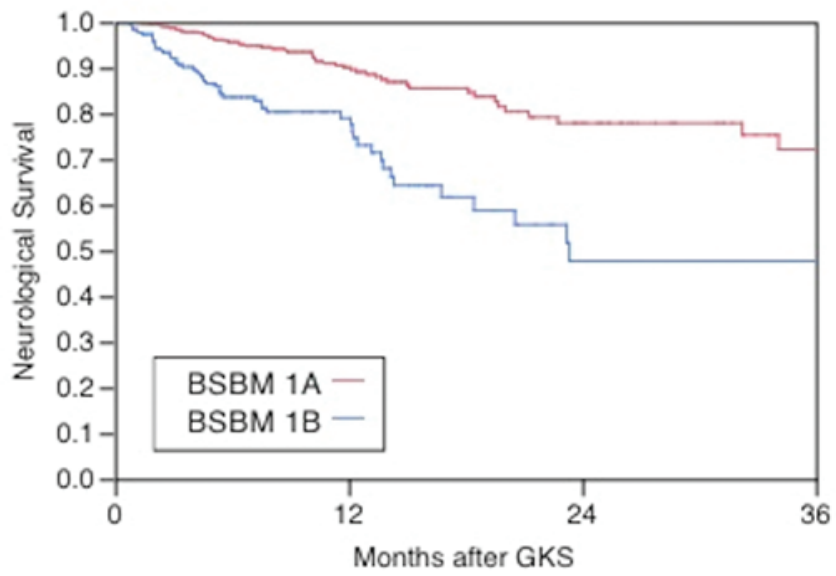

D. BSBM 3

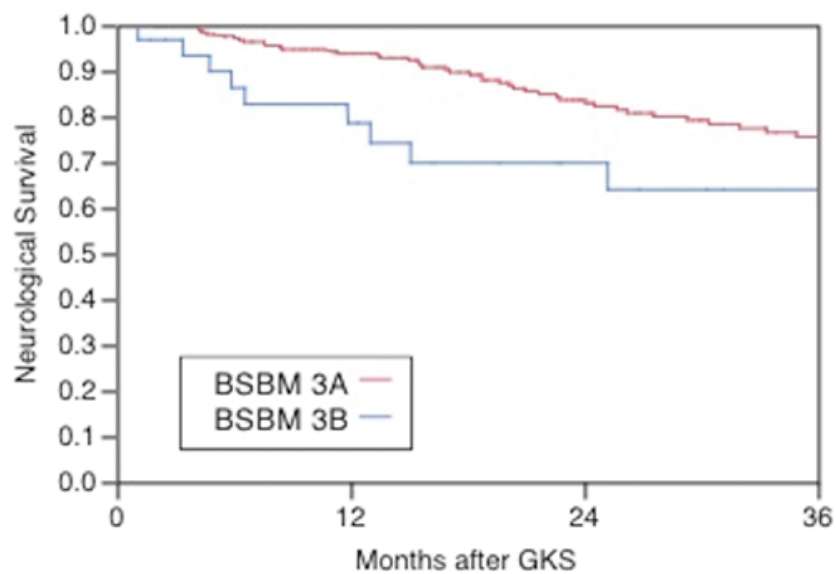

FIG. 5. Cumulative neurological death-free survival (neurological survival) curves, comparing NPS subgroups within the BSBM group. One-year neurological survival rates were $82.6 \%(0 \mathrm{~A}), 52.4 \%(0 \mathrm{~B}), 90.5 \%(1 \mathrm{~A}), 78.1 \%(1 \mathrm{~B}), 91.1 \%(2 \mathrm{~A}), 83.2 \%$ $(2 \mathrm{~B}), 93.9 \%(3 \mathrm{~A})$, and $76.3 \%(3 \mathrm{~B})$. Statistically significant differences in neurological survival were detected between all BSBM subgroups ( $O A$ vs $0 B, p=0.0001 ; 1 A$ vs $1 B, p<0.0001 ; 2 A$ vs $2 B, p=0.0002$; and $3 A$ vs $3 B, p=0.0052$ ).

extracranial diseases status, more aggressive radiation treatment should be considered. For such patients with $>10$ brain tumors and/or positive MRI findings of MD, combining WBRT with GKS is recommended. On the other hand, for those with neurological symptoms and/or total tumor volume $>15 \mathrm{~cm}^{3}$, staged GKS treatment with a 2- to 4-week interval or SRS is recommended. Thus, the modified BSBM enables us to decide the best GKS treatment strategy for various brain metastases by comparing neurological and systemic outcomes separately.
In general, the following indications for GKS are widely accepted: 1) up to 3 or 4 brain metastases, 2) tumor size $3 \mathrm{~cm}$ or less, 3) no MRI findings of MD, and 4) a KPS score $\geq 70 \%$. Despite our indications being somewhat more aggressive, neurological outcomes were satisfactory. We herein discuss indications for GKS from the viewpoint of NPS. The first parameter considered is tumor number. Recently, Serizawa et al. ${ }^{14}$ and Yamamoto et al. ${ }^{21}$ conducted a large prospective study (the JLGK0901 study) of 1200 patients. This study demonstrated that in

TABLE 6: Qualitative survival rates according to NPS in GPA*

\begin{tabular}{lccccc}
\hline \multicolumn{1}{c}{ GPA } & No. of Patients & SS-MST (mos) & NPS-A QS Rate at SS-MST (\%) & NPS-B QS Rate at SS-MST (\%) & $p$ Value \\
\hline $0-1.0$ & 1211 & 6.0 & 78.3 & 62.9 & $<0.0001$ \\
$1.5-2.5$ & 1429 & 11.4 & 87.1 & 69.5 & $<0.0001$ \\
3.0 & 153 & 36.9 & 90.4 & 50.8 & $<0.0001$ \\
$3.5-4.0$ & 45 & 45.7 & 97.4 & 80.0 & 0.0058 \\
\hline
\end{tabular}

* MST = median survival time; SS = systemic death-free survival. 
TABLE 7: Qualitative and neurological survival rates at the median systemic death-free survival time

\begin{tabular}{ccccc}
\hline Modified BSBM & No. of Patients & SS-MST (mos) & QS Rate at SS-MST (\%) & NS Rate at SS-MST (\%) \\
\hline OA & 204 & 2.6 & 96.4 & 98.6 \\
OB & 178 & 3.3 & 76.8 & 85.9 \\
1A & 825 & 6.2 & 92.0 & 96.2 \\
1B & 318 & 5.7 & 79.1 & 84.5 \\
2A & 792 & 14.0 & 83.0 & 88.3 \\
2B & 219 & 11.1 & 77.0 & 84.2 \\
3A & 268 & 33.3 & 69.9 & 77.8 \\
3B & 34 & 31.1 & 48.6 & 63.5 \\
\hline
\end{tabular}

terms of overall survival, GKS in patients with 5-10 brain metastases is not inferior to GKS in patients with 2-4 metastases, and it provided Level II evidence for GKSalone treatment for 1-10 brain metastases. ${ }^{21}$ Although there is no clinical evidence supporting GKS for $>10$ brain metastases, large retrospective reviews have revealed this treatment to be safe if the total tumor volume is $\leq 15-25 \mathrm{~cm}^{3}$ or the total skull absorption energy is $\leq$ 10-15 J. ${ }^{10,11,14,15,19}$ Considering the NPS, the presence of $>10$ brain metastases, even when associated with other poor neurological factors, is not a contraindication for GKS in BSBM 0 and 1 patients. BSBM 2A patients who have only 1 poor NPS such as $>10$ brain metastases (total tumor volume $\leq 15 \mathrm{~cm}^{3}$, no MRI findings of MD, and no neurological symptoms) appear to be good candidates for GKS-alone treatment.

The second parameter considered is tumor size. Generally, the local control rate decreases as tumor size increases..$^{10,13}$ We have been performing 3-staged GKS with a 2-week interval ${ }^{5}$ or 2-staged GKS with a 3- or 4-week interval. ${ }^{22}$ Using these techniques, we were able to decrease tumor recurrence and radiation injury. The third parameter considered is total tumor volume. We have advocated the 10-J total skull absorption energy concept to ensure the safety of Gamma Knife irradiation in a single session. ${ }^{13}$ Within these limits, none of our patients experienced acute brain swelling. If the total skull absorption energy exceeds $10 \mathrm{~J}$, we divide GKS into 2 or 3 sessions with less than $10 \mathrm{~J}$ per procedure. Multisession GKS or GKS combined with WBRT should be considered for BSBM 2 or 3 patients with large total tumor volumes.

The final parameter considered is MRI findings of MD. In general, we did not treat patients in whom obvious findings of nonlocalized MD involving more than a few gyri or sulci were shown on routine enhanced MRI (5-mm slice thickness) within 1 week before GKS treatment. However, when using 2-mm slice thickness 1.5-T MRI for dose planning, we occasionally recognized MDs involving one gyrus or sulcus. These localized MDs can often be controlled with GKS alone.

The modified BSBM system has a few limitations. Golden et al. $^{4}$ recommended the development of grading systems specific to primary lesions because favorable prognostic factors vary according to the original tumor. Recently, Sperduto et al. ${ }^{17}$ modified their original GPA system ${ }^{16}$ and developed a new index, the diagno- sis-specific GPA. Our new index, the modified BSBM, is only applicable to lung cancer, especially non-small cell lung cancer, because the majority of patients in this study $(56.5 \%, 1604$ patients) had non-small cell lung cancer. The modified BSBM failed to reach statistical significance for cancers of the gastrointestinal or urogenital tract and the breast, probably because the patient numbers of each BSBM class according to these cancer categories were too small. Furthermore, the recent development of molecular targeting agents will change survival durations, as well as neurological outcomes, if such agents are small enough to pass through the blood-brain barrier. In the future, we may need to establish a diagnosis-specific grading system that can predict life expectancy and neurological outcomes independently.

Our new modified BSBM appears to be uniquely useful for predicting neurological outcomes, such as preservation of neurological function and prevention of neurological death, independently of life expectancy in patients treated with GKS. Validity tests for other groups treated with GKS or other SRS devices are needed, and the modified BSBM should be considered when designing future clinical trials involving brain metastases treated by SRS, especially GKS. The cost of all SRS procedures for brain metastases are now reimbursed by the national health insurance program in Japan. In the future, this grading system may be used to authorize or deny such treatments for individual patients.

\section{Conclusions}

Our new grading system, the modified BSBM, can predict neurological consequences, such as maintenance of neurological function and prevention of neurological death, as well as life expectancy, independently of each other.

\section{Disclosure}

The authors report no conflict of interest concerning the materials or methods used in this study or the findings specified in this paper.

Author contributions to the study and manuscript preparation include the following. Conception and design: Serizawa, Higuchi, Nagano, Matsuda, Saeki, Miyakawa, Shibamoto. Acquisition of data: Serizawa, Nagano. Analysis and interpretation of data: Serizawa, Higuchi, Nagano, Matsuda, Miyakawa, Shibamoto. Drafting the article: Serizawa. Critically revising the article: Serizawa, 
Miyakawa, Shibamoto. Reviewed submitted version of manuscript: Serizawa, Miyakawa, Shibamoto. Approved the final version of the manuscript on behalf of all authors: Serizawa. Statistical analysis: Serizawa, Shibamoto. Administrative/technical/material support: Ono, Saeki, Shibamoto. Study supervision: Saeki, Hirai, Shibamoto.

\section{References}

1. Aoyama H, Shirato H, Tago M, Nakagawa K, Toyoda T, Hatano K, et al: Stereotactic radiosurgery plus whole-brain radiation therapy vs stereotactic radiosurgery alone for treatment of brain metastases: a randomized controlled trial. JAMA 295:2483-2491, 2006

2. Cox DR: Regression models and life tables. J R Stat Soc B Stat Methodol 34:187-220, 1972

3. Gaspar L, Scott C, Rotman M, Asbell S, Phillips T, Wasserman $\mathrm{T}$, et al: Recursive partitioning analysis (RPA) of prognostic factors in three Radiation Therapy Oncology Group (RTOG) brain metastases trials. Int J Radiat Oncol Biol Phys 37:745751,1997

4. Golden DW, Lamborn KR, McDermott MW, Kunwar S, Wara WM, Nakamura JL, et al: Prognostic factors and grading systems for overall survival in patients treated with radiosurgery for brain metastases: variation by primary site. J Neurosurg 109 Suppl:77-86, 2008

5. Higuchi Y, Serizawa T, Nagano O, Matsuda S, Ono J, Sato M, et al: Three-staged stereotactic radiotherapy without whole brain irradiation for large metastatic brain tumors. Int J Radiat Oncol Biol Phys 74:1543-1548, 2009

6. Kaplan EL, Meier P: Nonparametric estimation from incomplete observations. J Am Stat Assoc 53:457-481, 1958

7. Karnofsky DA, Buechenal JH: The clinical evaluation of chemotherapeutic agents in cancer, in MacLeod CM (ed): Evaluation of Chemotherapeutic Agent. New York: Columbia University Press, 1949, pp 191-205

8. Lorenzoni J, Devriendt D, Massager N, David P, Ruíz S, Vanderlinden B, et al: Radiosurgery for treatment of brain metastases: estimation of patient eligibility using three stratification systems. Int J Radiat Oncol Biol Phys 60:218-224, 2004

9. Serizawa T, Higuchi Y, Nagano O, Hirai T, Ono J, Saeki N, et al: Testing different brain metastasis grading systems in stereotactic radiosurgery: Radiation Therapy Oncology Group's RPA, SIR, BSBM, GPA, and modified RPA. Clinical article. J Neurosurg 117 Suppl:31-37, 2012

10. Serizawa T, Higuchi Y, Ono J, Matsuda S, Nagano O, Iwadate $\mathrm{Y}$, et al: Gamma Knife surgery for metastatic brain tumors without prophylactic whole-brain radiotherapy: results in 1000 consecutive cases. J Neurosurg 105 Suppl:86-90, 2006

11. Serizawa T, Hirai T, Nagano O, Higuchi Y, Matsuda S, Ono J, et al: Gamma knife surgery for 1-10 brain metastases without prophylactic whole-brain radiation therapy: analysis of cases meeting the Japanese prospective multi-institute study (JLGK0901) inclusion criteria. J Neurooncol 98:163-167, 2010

12. Serizawa T, Iuchi T, Ono J, Saeki N, Osato K, Odaki M, et al: Gamma knife treatment for multiple metastatic brain tumors compared with whole-brain radiation therapy. J Neurosurg 93 Suppl 3:32-36, 2000
13. Serizawa T, Saeki N, Higuchi Y, Ono J, Iuchi T, Nagano O, et al: Gamma knife surgery for brain metastases: indications for and limitations of a local treatment protocol. Acta Neurochir (Wien) 147:721-726, 2005

14. Serizawa T, Yamamoto M, Nagano O, Higuchi Y, Matsuda S, Ono J, et al: Gamma Knife surgery for metastatic brain tumors. A 2-institute study in Japan. J Neurosurg 109 Suppl: $118-121,2008$

15. Serizawa T, Yamamoto M, Sato Y, Higuchi Y, Nagano O, Kawabe T, et al: Gamma Knife surgery as sole treatment for multiple brain metastases: 2-center retrospective review of 1508 cases meeting the inclusion criteria of the JLGK0901 multiinstitutional prospective study. Clinical article. J Neurosurg 113 Suppl:48-52, 2010

16. Sperduto PW, Berkey B, Gaspar LE, Mehta M, Curran W: A new prognostic index and comparison to three other indices for patients with brain metastases: an analysis of $1,960 \mathrm{pa}-$ tients in the RTOG database. Int J Radiat Oncol Biol Phys 70:510-514, 2008

17. Sperduto PW, Chao ST, Sneed PK, Luo X, Suh J, Roberge D, et al: Diagnosis-specific prognostic factors, indexes, and treatment outcomes for patients with newly diagnosed brain metastases: a multi-institutional analysis of 4,259 patients. Int J Radiat Oncol Biol Phys 77:655-661, 2010

18. Weltman E, Salvajoli JV, Brandt RA, de Morais Hanriot R, Prisco FE, Cruz JC, et al: Radiosurgery for brain metastases: a score index for predicting prognosis. Int J Radiat Oncol Biol Phys 46:1155-1161, 2000

19. Yamamoto M, Ide M, Nishio Si, Urakawa Y: Gamma Knife radiosurgery for numerous brain metastases: is this a safe treatment? Int J Radiat Oncol Biol Phys 53:1279-1283, 2002

20. Yamamoto M, Serizawa T, Sato Y, Kawabe T, Higuchi Y, Nagano $\mathrm{O}$, et al: Validity of two recently-proposed prognostic grading indices for lung, gastro-intestinal, breast and renal cell cancer patients with radiosurgically-treated brain metastases. J Neurooncol 111:327-335, 2013

21. Yamamoto M, Serizawa T, Shuto T, Akabane A, Higuchi Y, Kawagishi J, et al: Stereotactic radiosurgery for patients with multiple brain metastases (JLGK0901): a multi-institutional prospective observational study. Lancet Oncol 15:387-395, 2014

22. Yomo S, Hayashi M, Nicholson C: A prospective pilot study of two-session Gamma Knife surgery for large metastatic brain tumors. J Neurooncol 109:159-165, 2012

Manuscript submitted May 6, 2014.

Accepted July 14, 2014.

Please include this information when citing this paper: DOI: 10.3171/2014.7.GKS14980.

Address correspondence to: Toru Serizawa, M.D., Ph.D., Tokyo Gamma Unit Center, Tsukiji Neurological Clinic, 1-9-9 Tsukiji, Chuou-ku, Tokyo 104-0045, Japan. email: gamma-knife.serizawa@ nifty.com. 